\title{
Abnormal dynamic ventilation function of COVID-19 survivors detected by pulmonary free-breathing proton MRI
}

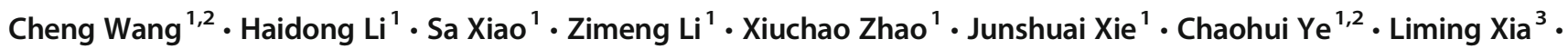 \\ Xin $\mathrm{Lou}^{4} \cdot$ Xin Zhou ${ }^{1}$
}

Received: 28 October 2021 / Revised: 13 December 2021 / Accepted: 22 January 2022 / Published online: 19 February 2022

(C) The Author(s), under exclusive licence to European Society of Radiology 2022

\begin{abstract}
Objectives To visualize and quantitatively assess regional lung function of survivors of COVID-19 who were hospitalized using pulmonary free-breathing ${ }^{1} \mathrm{H}$ MRI.

Methods A total of 12 healthy volunteers and 27 COVID-19 survivors ( $62.4 \pm 8.1$ days between infection and image acquisition) were recruited in this prospective study and performed chest ${ }^{1} \mathrm{H}$ MRI acquisitions with free tidal breathing. Then, conventional Fourier decomposition ventilation $(\mathrm{FD}-\mathrm{V})$ and global fractional ventilation $\left(\mathrm{FV}_{\mathrm{Global}}\right)$ were analyzed. Besides, a modified PREFUL (mPREFUL) method was developed to adapt to COVID-19 survivors and generate dynamic ventilation maps and parameters. All the ventilation maps and parameters were analyzed using Student's $t$-test. Pearson's correlation and a BlandAltman plot between $\mathrm{FV}_{\mathrm{Global}}$ and mPREFUL were analyzed.

Results There was no significant difference between COVID-19 and healthy groups regarding a static FD-V map $(0.47 \pm 0.12$ vs $0.42 \pm 0.08 ; p=.233$ ). However, mPREFUL demonstrated lots of regional high ventilation areas (high ventilation percentage (HVP): $23.7 \% \pm 10.6 \%$ ) existed in survivors. This regional heterogeneity (i.e., HVP) in survivors was significantly higher than in healthy volunteers $(p=.003)$. The survivors breathed deeper (flow-volume loop: $5375 \pm 3978$ vs $1688 \pm 789 ; p=.005$ ), and breathed more air in respiratory cycle (total amount: $62.6 \pm 19.3$ vs $37.3 \pm 9.9 ; p<.001$ ). Besides, mPREFUL showed both good Pearson's correlation $(r=0.74 ; p<.001)$ and Bland-Altman consistency (mean bias $=-0.01)$ with $\mathrm{FV}_{\mathrm{Global}}$.

Conclusions Dynamic ventilation imaging using pulmonary free-breathing ${ }^{1} \mathrm{H}$ MRI found regional abnormity of dynamic ventilation function in COVID-19 survivors.

Key Points

- Pulmonary free-breathing ${ }^{1} H$ MRI was used to visualize and quantitatively assess regional lung ventilation function of COVID19 survivors.

- Dynamic ventilation maps generated from ${ }^{1} H$ MRI were more sensitive to distinguish the COVID-19 and healthy groups (total air amount: $62.6 \pm 19.3$ vs $37.3 \pm 9.9 ; p<.001$ ), compared with static ventilation maps (FD-V value: $0.47 \pm 0.12$ vs $0.42 \pm$ $0.08 ; p=.233)$.

- COVID-19 survivors had larger regional heterogeneity (high ventilation percentage: $23.7 \% \pm 10.6 \%$ vs $13.1 \% \pm 7.9 \% ; p=$ .003), and breathed deeper (flow-volume loop: $5375 \pm 3978$ vs $1688 \pm 789 ; p=.005$ ) than healthy volunteers.
\end{abstract}

Cheng Wang and Haidong Li contributed equally to this work

Xin Zhou

xinzhou@wipm.ac.cn

1 State Key Laboratory of Magnetic Resonance and Atomic and Molecular Physics, National Center for Magnetic Resonance in Wuhan, Wuhan Institute of Physics and Mathematics, Innovation Academy for Precision Measurement Science and Technology, Chinese Academy of Sciences - Wuhan National Laboratory for Optoelectronics, Wuhan 430071, China
2 School of Physics, Huazhong University of Science and Technology, Wuhan 430074, China

3 Department of Radiology, Tongji Hospital, Tongji Medical College, Huazhong University of Science and Technology, Wuhan 430030, China

4 Department of Radiology, Chinese PLA General Hospital, Beijing 100853, China 
Keywords COVID-19 survivors · Pulmonary free-breathing ${ }^{1}$ H MRI $\cdot$ Dynamic ventilation imaging $\cdot$ Regional lung function . Phase-resolved functional lung (PREFUL)

\begin{tabular}{|c|c|}
\hline \multicolumn{2}{|c|}{ Abbreviations } \\
\hline End-exp & End-expiration \\
\hline End-insp & End-inspiration \\
\hline FD & Fourier decomposition \\
\hline FD-V & FD ventilation \\
\hline FRC & Full respiratory cycle \\
\hline FV & Fractional ventilation \\
\hline $\mathrm{FV}_{\text {Global }}$ & Global fractional ventilation \\
\hline HVP & High ventilation percentage \\
\hline PREFUL & Phase-resolved functional lung \\
\hline
\end{tabular}

\section{Introduction}

As of December 2021, more than 260 million people worldwide have been infected with coronavirus disease 2019 (COVID-19) [1, 2]. Recent studies indicated long-term lung function damage might exist among discharged COVID-19 survivors [3]. Previous studies on other coronavirus infections also showed persistent impairment on lung function would last for years [4]. Attention has been paid on assessment of COVID-19 survivors using pulmonary function tests (PFTs) [5-9]. However, PFTs have limited sensitivity to regional abnormalities, while regional lung function imaging could directly visualize function at regional and whole level [10].

Single-photon emission computed tomography (SPECT) is currently the gold standard of regional lung function imaging $[11,12]$. CT ventilation imaging (CTVI) [13] and its variant $\mathrm{N}$ Phase ventilation method [14] could also assess regional ventilation, and recently, CTVI was used to predict lung lesions in the early stage of COVID-19 disease [15]. Nevertheless, SPECT and CTVI are limited by radiation. Hyperpolarized gas magnetic resonance imaging (MRI) has also been used for functional imaging of lung [16-18], and our group detected damaged lung gas-exchange function with hyperpolarized ${ }^{129} \mathrm{Xe}$ MRI in COVID-19 survivors [19]. Hyperpolarized ${ }^{129}$ Xe MRI requires significant equipment and technical expertise and is thus currently not suitable for usage outside of expert centers.

Proton $\left({ }^{1} \mathrm{H}\right)$ MRI is one of the most widely used medical imaging modality [20, 21]. With development of fast acquisition and image processing, ${ }^{1} \mathrm{H}$ MRI could be used to assess regional lung function based on image registration or Fourier decomposition (FD) [22, 23]. Previous studies have shown ${ }^{1} \mathrm{H}$ MRI is a promising method for imaging lung ventilation function without radioactivity [24-32]. Howbeit, most methods do not visualize dynamic process of ventilation and might conceal some abnormal regions [33, 34]. Recently a phase-resolved functional lung (PREFUL) MRI method was proposed to quantify regional dynamic ventilation using pulmonary free- breathing ${ }^{1} \mathrm{H}$ MRI acquisition [35-39]. PREFUL MRI has been demonstrated to be more sensitive for detecting regional abnormity compared with static ventilation measurements.

Base on the above, we intend to visualize and quantitatively assess regional ventilation function of COVID-19 survivors using pulmonary free-breathing ${ }^{1} \mathrm{H}$ MRI. In this work, a modified PREFUL method (mPREFUL) is developed to adapt to COVID-19 survivors and generate dynamic ventilation maps and parameters. Besides, conventional FD ventilation (FD-V) [23] and global fractional ventilation $\left(\mathrm{FV}_{\mathrm{Global}}\right)[40]$ are also analyzed. We anticipate regional lung ventilation imaging using free-breathing ${ }^{1} \mathrm{H}$ MRI could help interpret the causes of lung dysfunction in COVID-19 survivors.

\section{Materials and methods}

\section{Study subjects}

A total of 12 healthy volunteers and 27 COVID-19 survivors were recruited in this prospective study. The local institutional review board approved the experiments and informed written consent was obtained from each subject. The survivors were discharged from Tongji Hospital, Wuhan, China, from February 4 to March 5, 2020. The inclusion criteria of survivors and healthy volunteers were as follows: (1) the survivors and healthy volunteers had no previous history of pulmonary disease or smoking; (2) the discharge time of survivors was about 1 month; (3) the age of healthy volunteers was close to the survivors as possible. One healthy volunteer and six COVID-19 survivors were excluded because of image quality (low SNR, motion artifacts, etc.). According to the guidelines of diagnosis of COVID-19 pneumonia [3, 41], the rest of the survivors were divided into mild and severe groups based on clinical symptoms during hospitalization (e.g., whether respiratory rate $>30$ breaths $/$ min or oxygen saturations $<$ $93 \%$ at rest state).

Thus, a total of 11 healthy volunteers (mean age \pm standard deviation (SD), $34 \pm 6$ years, and 5 females) and 21 COVID19 survivors ( $39 \pm 9$ years, 10 females, and divided into 13 mild and 8 severe survivors) were evaluated in this study. The images were acquired $62.4 \pm 8.1$ days later after the COVID infection. Among these survivors, 5 (23.8\%) had long COVID-19 symptoms ( 3 had dyspnea, 1 had chest pain, and 1 had palpitations). The remaining survivors had no obviously long COVID-19 symptoms (e.g., fatigue, cough). Demography characteristics of subjects are summarized in Table 1. 
Table 1 The demography and pulmonary function characteristics of all the subjects

\begin{tabular}{llllll}
\hline \multirow{2}{*}{ Characteristics } & Healthy $(n=11)$ & \multicolumn{2}{l}{ COVID-19 $(n=21)$} & \multirow{2}{*}{$p$ value $^{\text {a }}$} \\
\cline { 3 - 5 } & & Mild $(n=13)$ & Severe $(n=8)$ & All $(n=21)$ & \\
\hline Age (years) & $34 \pm 6$ & $36 \pm 9$ & $44 \pm 8$ & $39 \pm 9$ & .101 \\
Female & $5(45.5 \%)$ & $9(69.2 \%)$ & $1(12.5 \%)$ & $10(47.6 \%)$ & .911 \\
$\mathrm{FEV}_{1} / \mathrm{FVC}(\%)$ & $80.9 \pm 4.1$ & $81.7 \pm 2.6$ & $86.4 \pm 4.9$ & $83.2 \pm 4.0$ & .137 \\
$\mathrm{PaO}_{2}$ (mmHg) & $98.4 \pm 0.7$ & $98.4 \pm 0.9$ & $98.5 \pm 0.8$ & $98.4 \pm 0.8$ & .848 \\
$\mathrm{Hospital} \mathrm{stay} \mathrm{(days)}$ & N/A & $23.2 \pm 7.2$ & $28.3 \pm 7.9$ & $25.3 \pm 7.7$ & N/A \\
Discharge time (days) & N/A & $35.1 \pm 6.1$ & $41.3 \pm 13.8$ & $37.4 \pm 9.8$ & N/A \\
Infection to image & N/A & $58.3 \pm 3.4$ & $69.6 \pm 9.0$ & $62.4 \pm 8.1$ & N/A \\
$\quad$ acquisition (days) & N/A & $2(15.4 \%)$ & $1(12.5 \%)$ & $3(14.3 \%)$ & N/A \\
Dyspnea & N/A & $0(0 \%)$ & $1(12.5 \%)$ & $1(4.8 \%)$ & N/A \\
Chest pain & N/A & $0(0 \%)$ & $1(12.5 \%)$ & $1(4.8 \%)$ & N/A \\
Palpitations & & &
\end{tabular}

Values were presented as mean $\pm \mathrm{SD}$ or $n(\%)$

${ }^{a}$ The significant difference between the healthy volunteers and all the COVID-19 survivors was determined with Student's $t$-test

COVID-19, corona virus disease 2019; $F E V_{1} / F V C$, the ratio of forced expiratory volume in first second and forced vital capacity; $\mathrm{PaO}_{2}$, arterial partial pressure of oxygen

\section{Image acquisitions}

Pulmonary free-breathing ${ }^{1} \mathrm{H}$ MRI images were acquired from all subjects using a 3-T scanner (uMR 780, United Imaging Healthcare) in head-first supine position. One coronal slice at the middle of lungs was acquired using a chest coil. The acquisitions were performed during free tidal breathing using a spoiled gradient echo sequence over a period of $85 \mathrm{~s}$ at a temporal resolution of $0.441 \mathrm{~s}$ (total 192 time-series ${ }^{1} \mathrm{H}$ MRI images for each subject). The sequence settings were field of view $50 \times 50 \mathrm{~cm}^{2}$, matrix size $256 \times 256$, slice thickness 15 $\mathrm{mm}$, echo time $1.04 \mathrm{~ms}$, repetition time $3 \mathrm{~ms}$, flip angle $5^{\circ}$, and bandwidth $384 \mathrm{kHz}(1500 \mathrm{~Hz} /$ pixel $\times 256$ pixel $)$.

\section{Dynamic ventilation imaging}

The acquired time-series ${ }^{1} \mathrm{H}$ MRI images were pre-processed (e.g., first three images omitted, de-noising, lung segmentation) and spatially co-registered [23]. Then, dynamic ventilation maps were generated by using mPREFUL. The general framework of mPREFUL included three major steps: (1) timeseries signal construction, (2) full respiratory cycle (FRC) reconstruction, and (3) dynamic ventilation maps generation, as shown in Fig. 1. Compared with original PREFUL [35], an automatic method was used to construct time-series signal in mPREFUL, and thus the dynamic ventilation maps could be automatically generated. Besides, the actual respiratory cycle time in FRC of each subject was calculated in mPREFUL. Correspondingly, the dynamic ventilation imaging of subjects was assessed based on their real respiratory time. The detailed description of mPREFUL is provided in the Supplementary Material.

\section{Dynamic ventilation parameters}

In mPREFUL, the dynamic ${ }^{1} \mathrm{H}$-density difference maps [22] and relative difference maps [26] between each time point $\left(t_{\mathrm{x}}\right)$ ${ }^{1} \mathrm{H}$ MRI image and end-exp ${ }^{1} \mathrm{H}$ MRI image in FRC were used to represent the dynamic ventilation maps (denoted as $\mathrm{V}\left(t_{\mathrm{x}}\right)$ maps) and dynamic fractional ventilation maps (denoted as $\mathrm{FV}\left(t_{\mathrm{x}}\right)$ maps). The mean value of a time point $\mathrm{V}\left(t_{\mathrm{x}}\right)$ map/ $\mathrm{FV}\left(t_{\mathrm{x}}\right)$ map was calculated to reflect the air volume/relative air volume that was breathed in that time point. Then, these time-varying scattered points were fitted into a continuous curve, denoted as $\mathrm{V}\left(t_{\mathrm{x}}\right)$ curve or $\mathrm{FV}\left(t_{\mathrm{x}}\right)$ curve. The $\mathrm{V}\left(t_{\mathrm{x}}\right)$ curve and $\mathrm{FV}\left(t_{\mathrm{x}}\right)$ curve directly reflected dynamic change of air amount that was breathed during respiratory cycle. The slopes of $\mathrm{V}\left(t_{\mathrm{x}}\right)$ curve $/ \mathrm{FV}\left(t_{\mathrm{x}}\right)$ curve reflected the air flow rate (i.e., the air amount that was breathed per unit time). Specially, the endinsp $\mathrm{V}\left(t_{\mathrm{x}}\right)$ map and $\mathrm{FV}\left(t_{\mathrm{x}}\right)$ map were referred as $\mathrm{V}_{100 \%}$ map and $\mathrm{FV}_{100 \%}$ map, and the mean value of $\mathrm{V}_{100 \%} / \mathrm{FV}_{100 \%}$ map reflected the total air volume/relative air volume that was breathed in whole respiratory cycle. The $\mathrm{V}\left(t_{\mathrm{x}}\right)$ curve and its slopes could be plotted as a closed air flow-volume loop (as displayed in Figure 3), which was used to reflect the breathing state during free tidal breathing [42]. In addition, the regional heterogeneity marker of dynamic ventilation maps was generated from the $K$-means cluster which was commonly used in the post-processing of ventilation imaging [43]. 


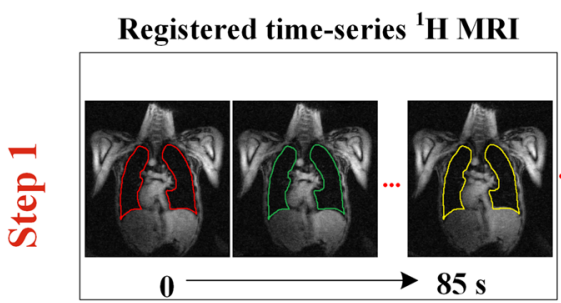

Acquisition time

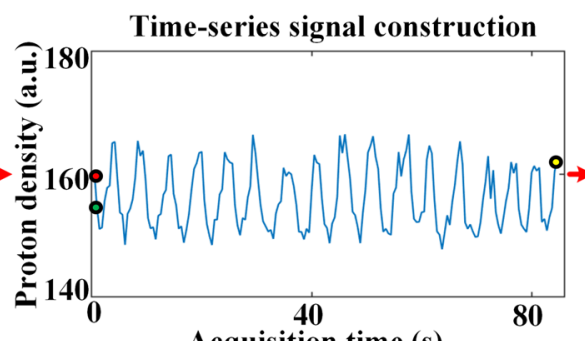

Acquisition time (s)
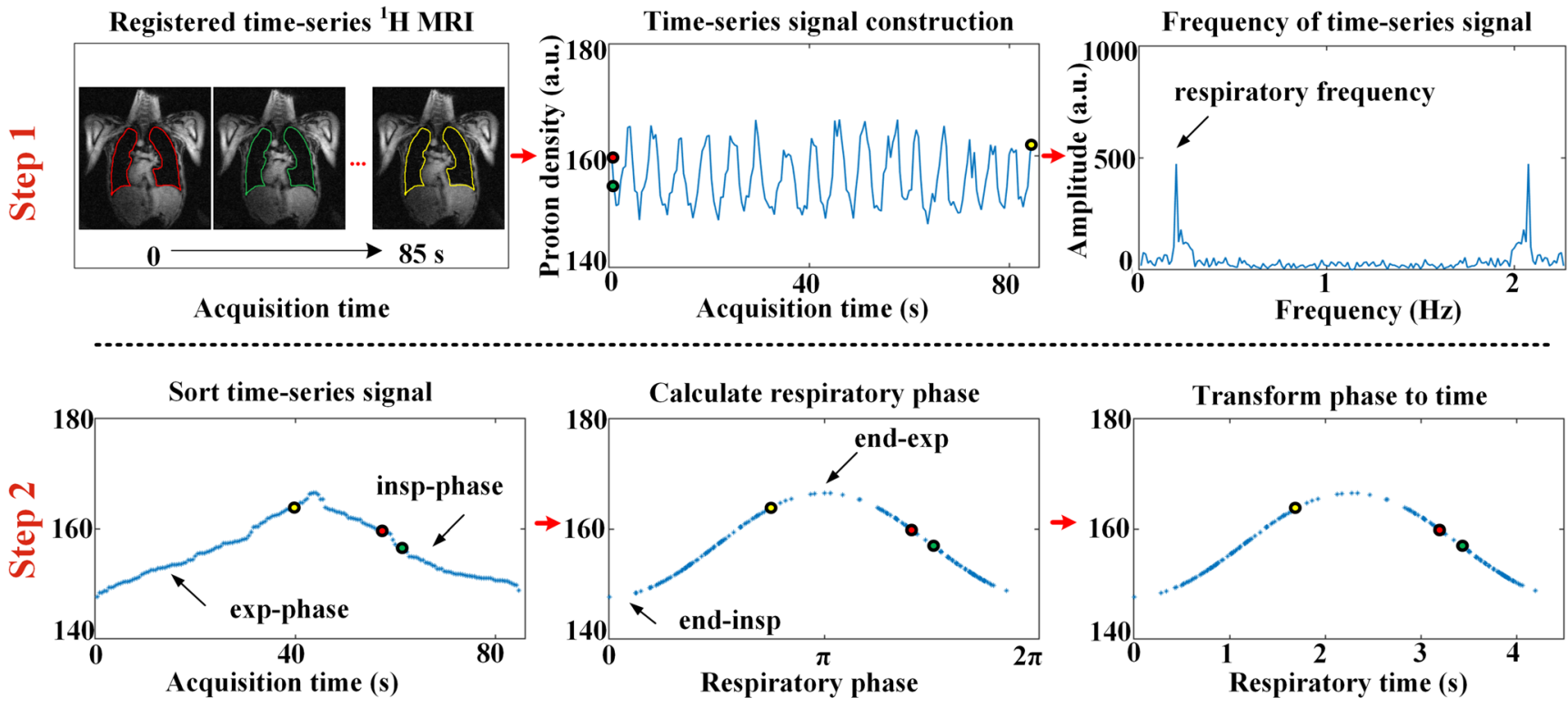

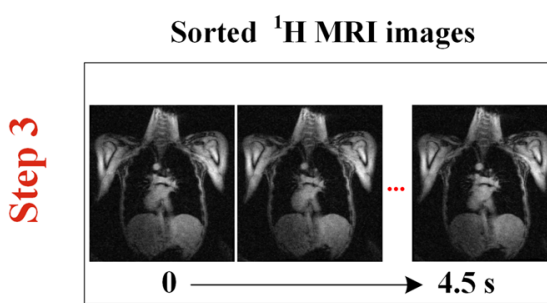

Respiratory cycle
Dynamic ${ }^{1} \mathrm{H}$-density difference maps

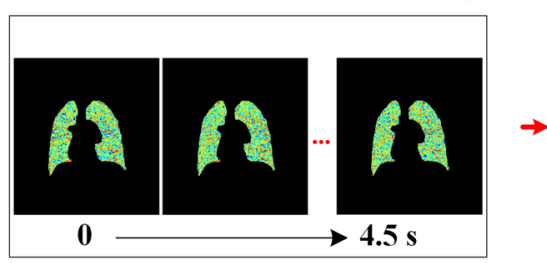

Respiratory cycle
Dynamic ventilation parameters

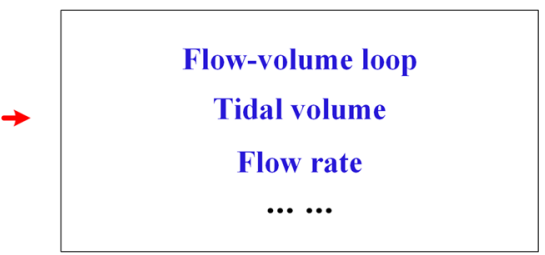

-....
Fig. 1 The framework of mPREFUL by using free-breathing ${ }^{1} \mathrm{H}$ MRI, which contained three steps. Step 1 served to construct time-series signal according to the mean values of lung proton density. Step 2 served to sort the time-series signal in exp-phase and insp-phase of the full respiratory cycle (FRC), and then calculate the respiratory phase and respiratory time of each image according to the cosine model. Step 3 served to calculate the dynamic ${ }^{1} \mathrm{H}$-density difference and relative difference maps between each time point image and the end-exp image in the FRC. Note that the proton density was expressed in arbitrary units (a.u.)

\section{Validation of mPREFUL}

The end-exp and end-insp lung volumes of the free-breathing ${ }^{1} \mathrm{H}$ MRI images were manually extracted and segmented by two radiologists (with more than 10 years of experience in pulmonary imaging diagnosis) [40]. The relative change of end-exp and end-insp lung volumes was calculated and denoted as $\mathrm{FV}_{\text {Global }}$ [40]. The $\mathrm{FV}_{\text {Global }}$ value reflected the relative air volume that was breathed in whole respiratory cycle and could be used to verify the ventilation maps generated from mPREFUL [22, 28, 40].

\section{Statistical analysis}

The FD-V maps [23] of the subjects were used as static ventilation maps [36]. To correct out-of-phase ventilation, the N-Phase ventilation method [14] was used to obtain the adjusted dynamic ventilation maps. All the ventilation maps and parameters of different groups were analyzed using unpaired 2-tailed Student's $t$-test. Pearson's correlation and a Bland-Altman plot between mPREFUL and $\mathrm{FV}_{\mathrm{Global}}$ results were analyzed. All the statistical analyses were performed on SPSS statistics software (version 22.0, IBM Inc.). Significance was determined by using a difference with $p<0.05$.

\section{Results}

\section{Dynamic ventilation maps}

Figure 2 shows the dynamic ${ }^{1} \mathrm{H}$ MRI images (at endexp, middle, and end-insp time points) and corresponding dynamic ventilation maps (at middle and end-insp, i.e., $\mathrm{V}_{50 \%}$ map and $\mathrm{V}_{100 \%}$ map) of a healthy volunteer (male, 31 years old), a mild COVID-19 survivor (female, 47 years old), and a severe COVID-19 survivor (male, 53 years old). The green and red lines denoted 

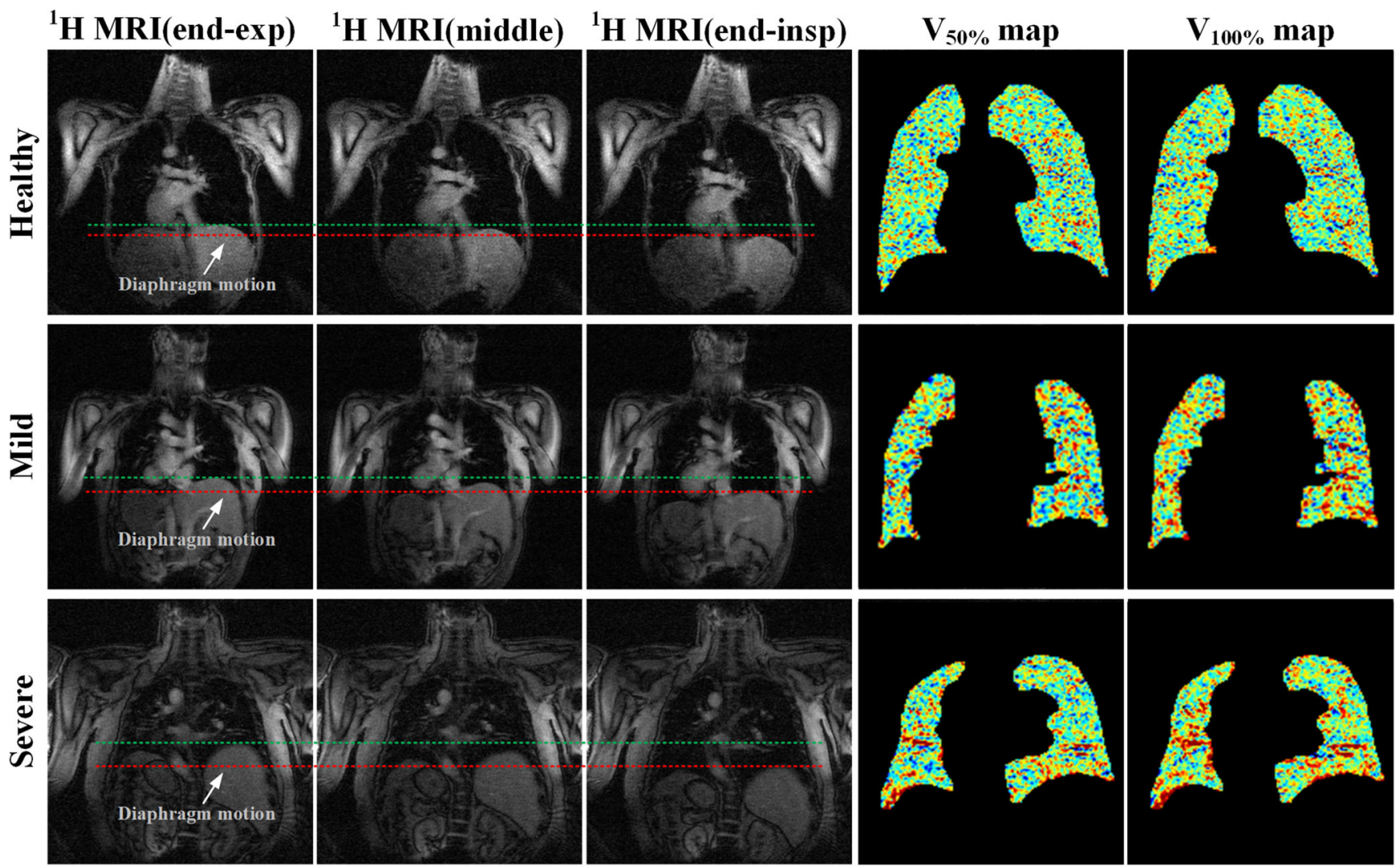

low ventilation $=$ high ventilation

Fig. 2 The dynamic ${ }^{1} \mathrm{H}$ MRI images at the end-exp, middle, and end-insp time points, and corresponding dynamic ventilation maps $\left(\mathrm{V}\left(t_{\mathrm{x}}\right)\right.$ maps) at the middle time point $\left(\mathrm{V}_{50 \%}\right.$ map) and end-insp time point $\left(\mathrm{V}_{100 \%}\right.$ map $)$ of a healthy volunteer (male, 31 years old), a mild COVID-19 survivor (female, 47 years old), and a severe COVID-19 survivor (male, 53 years old). The distance between green line and red line in dynamic ${ }^{1} \mathrm{H}$ MRI images indicated the range of diaphragm motion in the respiratory cycle. The hyperintense signal areas (red color areas) of the $\mathrm{V}_{50 \%}$ map and $\mathrm{V}_{100 \%}$ map indicated high ventilation areas

the adjusted $\mathrm{V}_{100 \%}$ maps by N-Phase ventilation method are shown in Fig. S2 of the Supplementary Material.

\section{Dynamic ventilation parameters}

Figure 3 shows the $\mathrm{V}\left(t_{\mathrm{x}}\right)$ curves, flow-volume loops, and $\mathrm{FV}\left(t_{\mathrm{x}}\right)$ curves of a healthy volunteer (male, 31 years old), a mild COVID-19 survivor (female, 47 years old), and a severe COVID-19 survivor (male, 38 years old). The $\mathrm{V}\left(t_{\mathrm{x}}\right)$ curve in healthy volunteer was relatively flatter than that in survivors. Correspondingly, the slope of the $\mathrm{V}\left(t_{\mathrm{x}}\right)$ curve in survivors was larger, indicating survivors breathed more air per unit time. The peak value of $\mathrm{V}\left(t_{\mathrm{x}}\right)$ curve (i.e., the mean value of $\mathrm{V}_{100 \%}$ map) in survivors was larger, indicating survivors breathed more air in respiratory cycle. The flow-volume loops in survivors were larger, reflecting survivors had a deep breathing state during respiratory cycle. The slope and peak value (i.e., the mean value of $\mathrm{FV}_{100 \%}$ map) of $\mathrm{FV}\left(t_{\mathrm{x}}\right)$ curves in survivors were also larger than those in healthy volunteer, also 

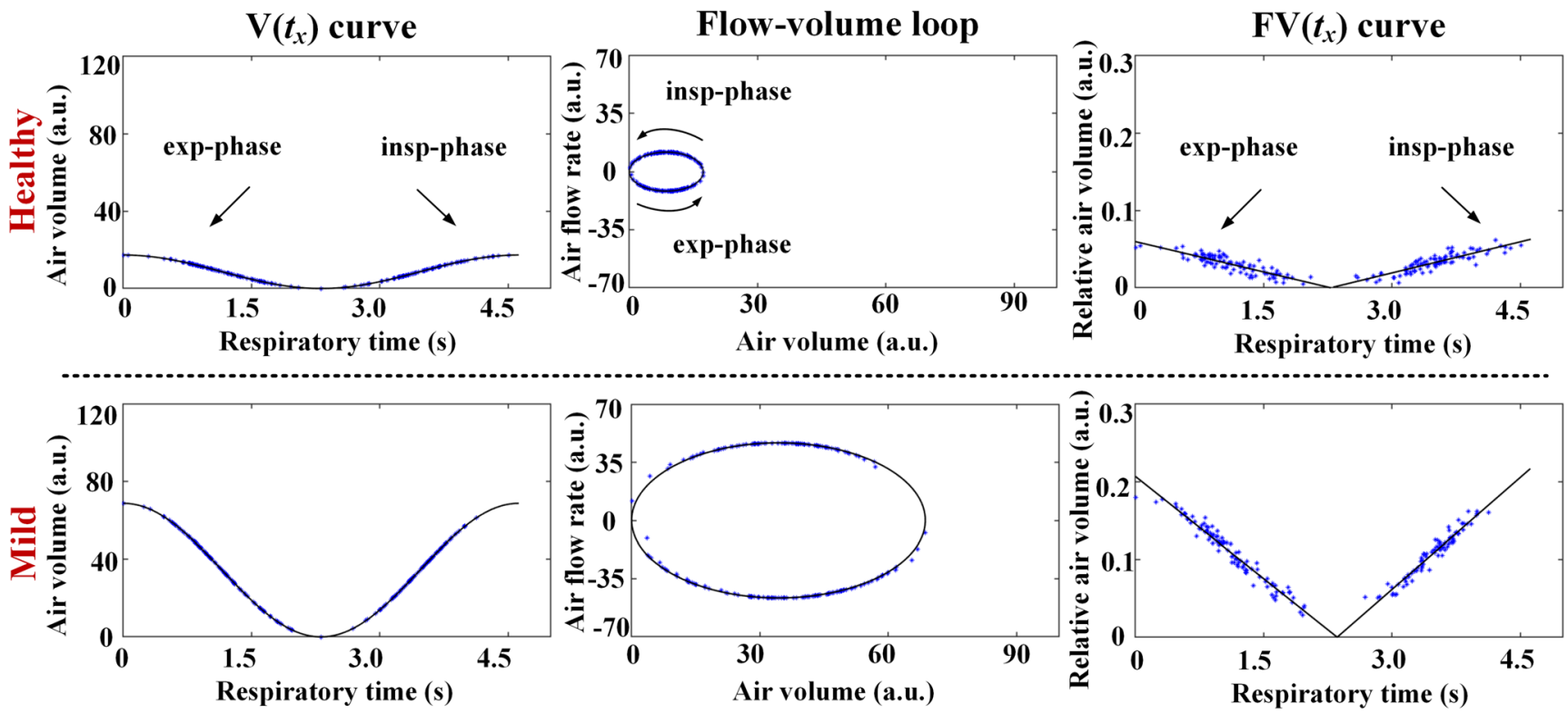
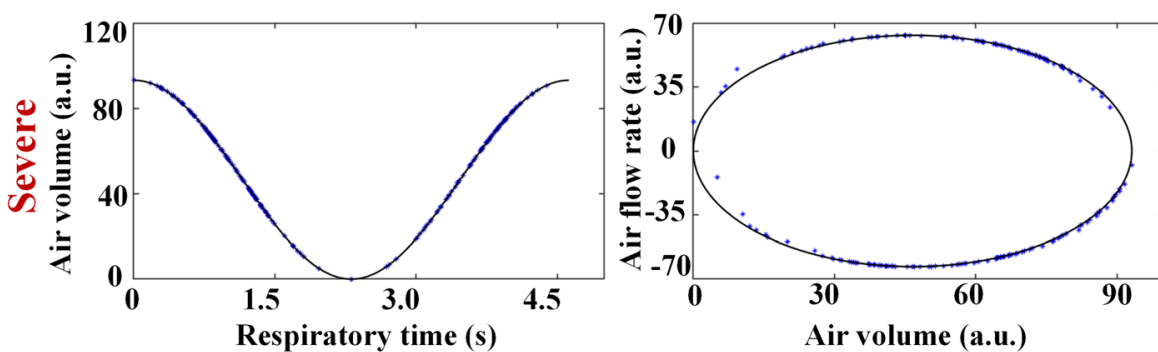

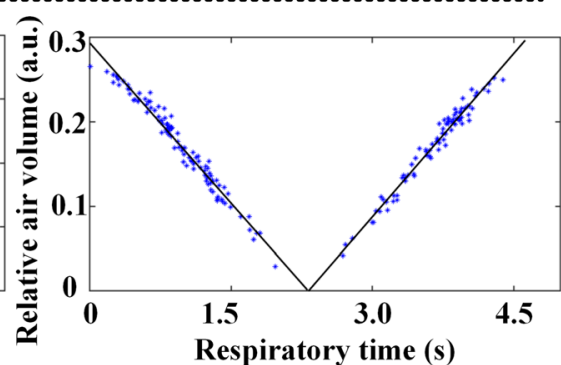

Respiratory time (s)
Fig. 3 The $\mathrm{V}\left(t_{\mathrm{x}}\right)$ curves, flow-volume loops, and $\mathrm{FV}\left(t_{\mathrm{x}}\right)$ curves of a healthy volunteer (male, 31 years old), a mild COVID-19 survivor (female, 47 years old), and a severe COVID-19 survivor (male, 38 years old). In $\mathrm{V}\left(t_{\mathrm{x}}\right)$ curves, the blue points represented the mean values of $\mathrm{V}\left(t_{\mathrm{x}}\right)$ maps at each time point, which were then fitted into continuous curves (black solid curves). In flow-volume loops, the blue points represented the slopes of $\mathrm{V}\left(t_{\mathrm{x}}\right)$ curves at each time point. In $\mathrm{FV}\left(t_{\mathrm{x}}\right)$ curves, the blue points represented the mean values of $\mathrm{FV}\left(t_{\mathrm{x}}\right)$ maps at each time point indicating survivors breathed more air per unit time and breathed more air in respiratory cycle.

\section{Regional dynamic ventilation}

Figure 4 shows the regional heterogeneity analysis of the dynamic ventilation maps ( $\mathrm{V}_{100 \%}$ maps) of the representative subjects in Fig. 2. The lung pixels of $\mathrm{V}_{100 \%}$ maps were categorized as normal ventilation regions (green color) and high ventilation regions (red color) by $K$-means cluster. It could be seen that the high ventilation regions in healthy volunteer were uniformly distributed in lungs. However, in survivors, the high ventilation regions were heterogeneous and aggregated in some regions. The high ventilation percentage (HVP) of the lungs in survivors was higher than that in healthy volunteer. Besides, the regional $\mathrm{V}\left(t_{\mathrm{x}}\right)$ curves of normal and high ventilation regions were also displayed. The slope and peak value in healthy volunteer were small. While in survivors, the regional $\mathrm{V}\left(t_{\mathrm{x}}\right)$ curves of normal ventilation regions were similar with that of healthy volunteer, but the regional $\mathrm{V}\left(t_{\mathrm{x}}\right)$ curves of high ventilation regions were obviously different with a large slope and peak value. In this way, the regional dynamic ventilation information of the subjects could be provided and quantitatively assessed.

\section{Group analysis}

Table 2 and Figure 5 display group analysis of the static FD-V map, dynamic ventilation maps ( $\mathrm{V}_{100 \%}$ map, $\mathrm{FV}_{100 \%}$ map), dynamic ventilation parameters (HVP, $\mathrm{V}\left(t_{\mathrm{x}}\right)$ curve, $\mathrm{FV}\left(t_{\mathrm{x}}\right)$ curve, and flow-volume loop), and $\mathrm{FV}_{\mathrm{Global}}$. There was no significant difference between the COVID-19 and healthy groups regarding mean value of $F D-V$ map $(0.47 \pm 0.12$ vs $0.42 \pm$ $0.08 ; p=0.233$ ). However, dynamic ventilation maps and parameters all showed significant difference between survivors and healthy volunteers. The mean value of $\mathrm{V}_{100 \%}$ map/ $\mathrm{FV}_{100 \%}$ map in survivors was significantly higher than in healthy volunteers $(62.6 \pm 19.3 / 15.1 \% \pm 4.1 \%$ vs $37.3 \pm 9.9 / 9.2 \% \pm 2.1 \% ; p<0.001 / p<0.001)$. The slope of $\mathrm{V}\left(t_{\mathrm{x}}\right)$ curve/FV $\left(t_{\mathrm{x}}\right)$ curve in survivors was significantly higher than in healthy volunteers $(43.6 \pm 17.0 / 8.9 \% \pm$ 


\section{Regional heterogeneity}
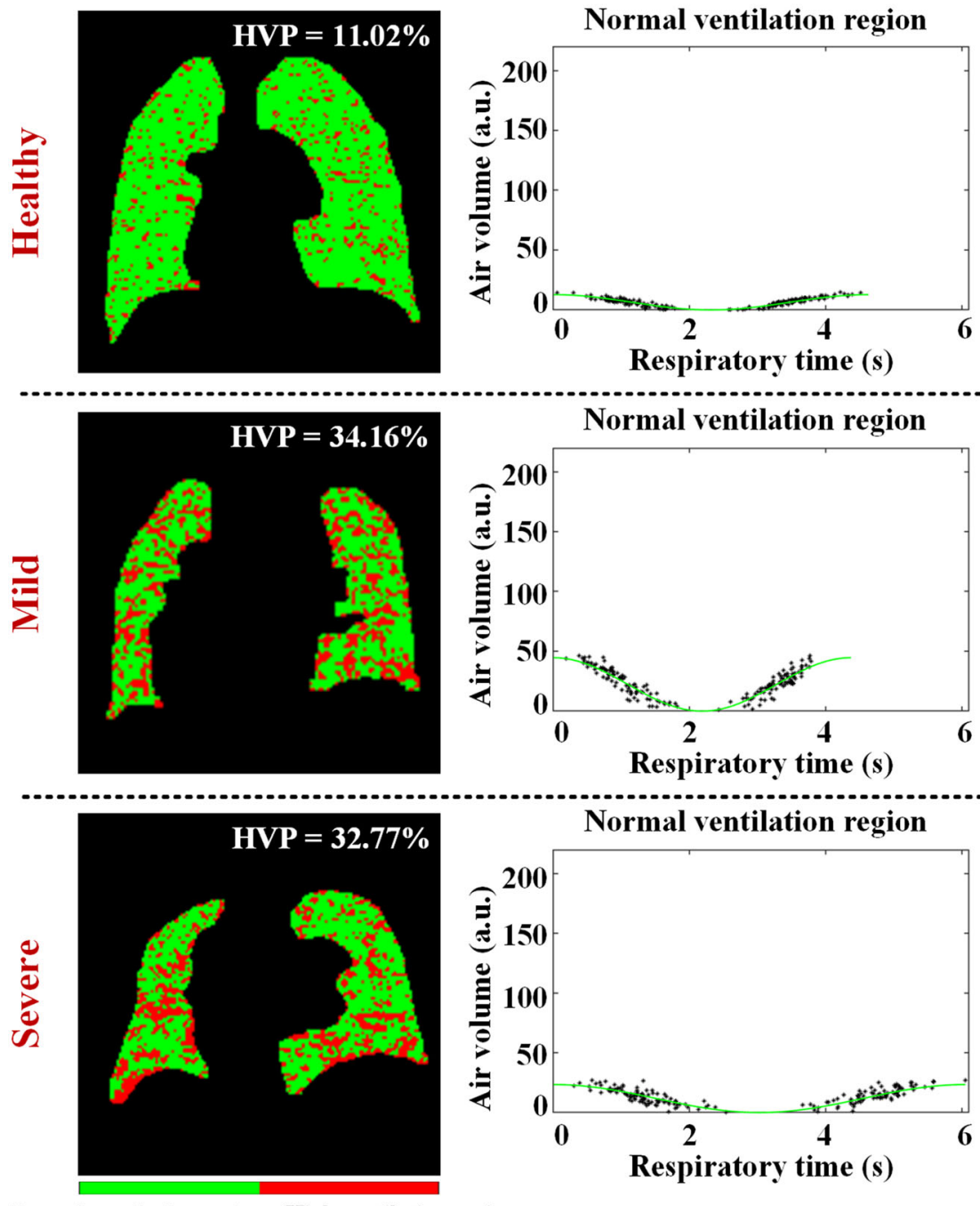

Normal ventilation region

Normal ventilation region High ventilation region (green areas) (red areas)

Fig. 4 The regional heterogeneity analysis of dynamic ventilation maps $\left(\mathrm{V}_{100 \%}\right.$ map) of the representative subjects (the same subjects in Fig. 2), and the regional $\mathrm{V}\left(t_{\mathrm{x}}\right)$ curves of these subjects. The green areas in lungs represented the normal ventilation regions, and the aggregate of all red

$2.9 \%$ vs $24.3 \pm 7.3 / 5.2 \% \pm 1.4 \% ; p=0.001 / p<0.001)$. The area of flow-volume loop in survivors was significantly higher than in healthy volunteers $(5375 \pm 3978$ vs $1688 \pm 789 ; p=0.005)$. Besides, the $\mathrm{FV}_{\text {Global }}$ in survivors was also significantly higher than in healthy volunteers $(12.9 \% \pm 3.9 \%$ vs $8.8 \% \pm 1.9 \% ; p=0.003)$.

Specially, the regional heterogeneity marker (HVP) in the lungs of survivors was significantly higher than in healthy volunteers $(23.7 \% \pm 10.6 \%$ vs $13.1 \% \pm 7.9 \% ; p=0.003)$. The HVP in 10 of $11(90.9 \%)$ healthy volunteers were belonging to the range $(5.8 \%, 15.9 \%)$, while only 7 of 21 (33.3\%) survivors have HVP values in that range.

\section{Regional V $\left(t_{x}\right)$ curve}
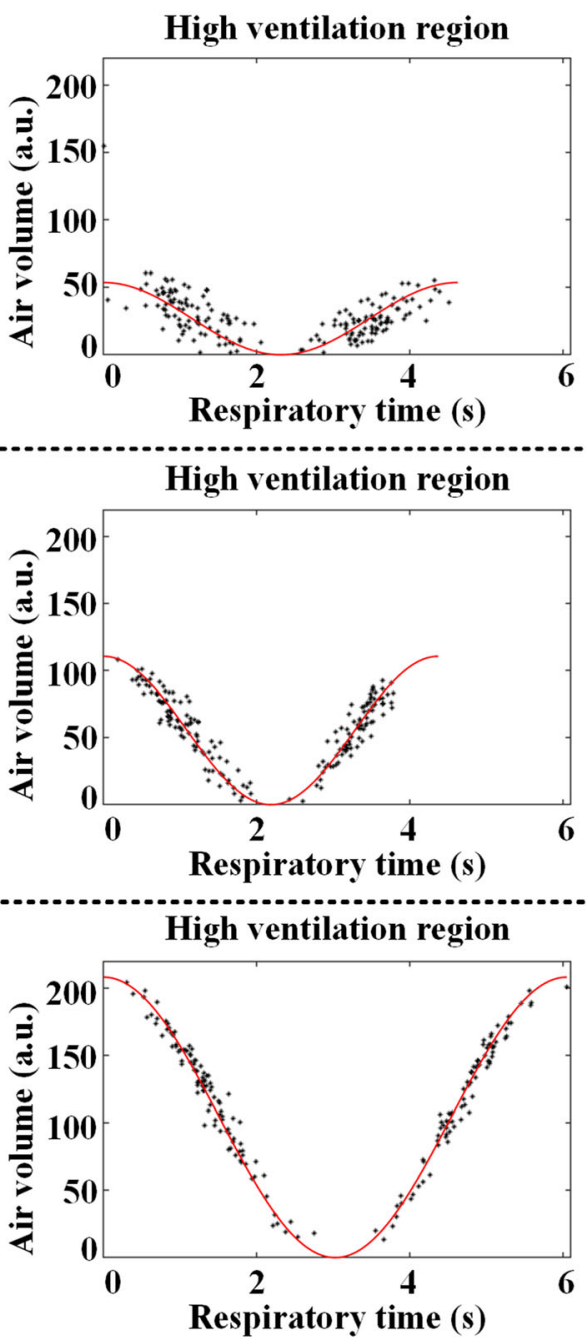

areas in the heterogeneity maps represented the high ventilation regions. The high ventilation percentage (HVP) of the lungs was labeled in these subjects

\section{Validation of $m$ PREFUL}

Figure 6a shows the validation of the dynamic ventilation map ( $\mathrm{FV}_{100 \%}$ maps) by measuring the relative changes between the end-exp lung volume and end-insp lung volume of the freebreathing ${ }^{1} \mathrm{H}$ MRI images of all subjects. The measured relative changes of lung volumes and the calculated mean $\mathrm{FV}$ values of $\mathrm{FV}_{100 \%}$ maps of all subjects were $0.11 \pm 0.04$ and $0.13 \pm 0.04$, without significant difference $(p=0.142)$. Figure $6 \mathrm{~b}$ shows Pearson's correlations between the measured relative changes and the calculated mean $\mathrm{FV}$ values. The two methods had a good correlation $(r=0.74 ; p<0.001)$. Figure $6 \mathrm{c}$ shows a BlandAltman plot of two methods, where mean bias \pm SD was 
Table 2 The group results (mean $\pm \mathrm{SD}$ ) of static FD-V map (mean value), dynamic ventilation maps (mean value of $\mathrm{V}_{100 \%}$ map and mean value of $\mathrm{FV}_{100 \%}$ map), dynamic ventilation parameters (HVP, slope of
$\mathrm{V}\left(t_{\mathrm{x}}\right)$ curve, slope of $\mathrm{FV}\left(t_{\mathrm{x}}\right)$ curve, and area of flow-volume loop), and $\mathrm{FV}_{\mathrm{Global}}$ for the healthy volunteers, mild COVID-19 survivors, severe COVID-19 survivors, and all the COVID-19 survivors

\begin{tabular}{|c|c|c|c|c|c|c|c|c|}
\hline Group & FD-V map & $\mathrm{V}_{100 \%}$ map & $\mathrm{FV}_{100 \%}$ map & HVP & $\mathrm{V}\left(t_{\mathrm{x}}\right)$ curve & $\mathrm{FV}\left(t_{\mathrm{x}}\right)$ curve & Flow-volume loop & $\mathrm{FV}_{\text {Global }}$ \\
\hline Healthy & $0.42 \pm 0.08$ & $37.3 \pm 9.9$ & $9.2 \% \pm 2.1 \%$ & $13.1 \% \pm 7.9 \%$ & $24.3 \pm 7.3$ & $5.2 \% \pm 1.4 \%$ & $1688 \pm 789$ & $8.8 \% \pm 1.9 \%$ \\
\hline Mild & $0.48 \pm 0.14$ & $57.9 \pm 15.7$ & $14.7 \% \pm 3.1 \%$ & $21.6 \% \pm 10.5 \%$ & $37.9 \pm 9.9$ & $8.2 \% \pm 2.2 \%$ & $4094 \pm 1966$ & $12.0 \% \pm 3.5 \%$ \\
\hline Severe & $0.45 \pm 0.09$ & $70.1 \pm 23.0$ & $15.7 \% \pm 5.5 \%$ & $27.1 \% \pm 10.5 \%$ & $52.9 \pm 22.4$ & $10.1 \% \pm 3.6 \%$ & $7455 \pm 5532$ & $14.3 \% \pm 4.5 \%$ \\
\hline COVID-19 & $0.47 \pm 0.12$ & $62.6 \pm 19.3$ & $15.1 \% \pm 4.1 \%$ & $23.7 \% \pm 10.6 \%$ & $43.6 \pm 17.0$ & $8.9 \% \pm 2.9 \%$ & $5375 \pm 3978$ & $12.9 \% \pm 3.9 \%$ \\
\hline$P_{\mathrm{H}-\mathrm{M}}$ & .208 & .001 & $<.001$ & .037 & .001 & .001 & .001 & .011 \\
\hline$P_{\mathrm{H}-\mathrm{S}}$ & .456 & .001 & .002 & .004 & .001 & .001 & .003 & .002 \\
\hline$P_{\mathrm{M}-\mathrm{S}}$ & .556 & .164 & .605 & .263 & .048 & .147 & .058 & .194 \\
\hline$P_{\mathrm{H}-\mathrm{C}}$ & .233 & $<.001$ & $<.001$ & .003 & .001 & $<.001$ & .005 & .003 \\
\hline
\end{tabular}

$F D-V$, Fourier decomposition ventilation; $H V P$, high ventilation percentage; $F V_{G l o b a l}$, global fractional ventilation; $C O V I D-19$, corona virus disease 2019; $P_{H-M}, p$ value between healthy volunteers and mild survivors; $P_{H-S}, p$ value between healthy volunteers and severe survivors; $P_{M-S}, p$ value between mild survivors and severe survivors; $P_{H-C}, p$ value between healthy volunteers and all the COVID-19 survivors

$-0.01 \pm 0.03$ (95\% limit of agreement: -0.07 to 0.04$)$. Besides, the comparisons between FV and lung volumes (e.g., functional residual capacity, tidal volume) are shown in Fig. S3 of the Supplementary Material. These results indicated the measured relative changes and the calculated mean $\mathrm{FV}$ values were in good agreement, and supported the accuracy of the proposed method.

\section{Discussion}

In this study, pulmonary free-breathing ${ }^{1} \mathrm{H}$ MRI was used to visualize and quantitatively assess regional
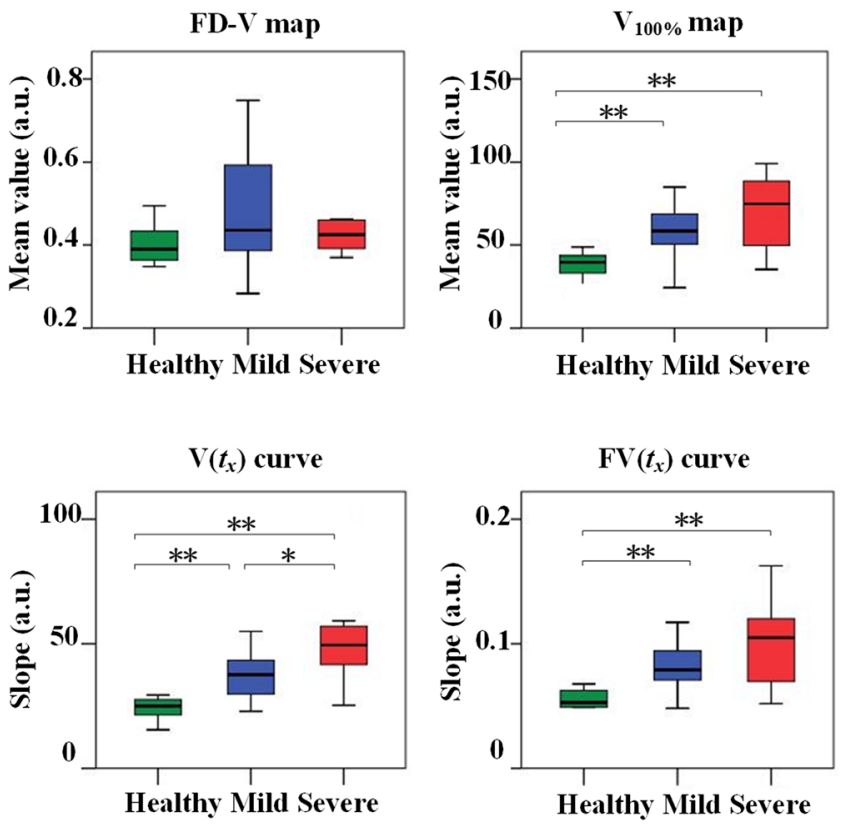

Fig. 5 The group analysis of FD-V map, dynamic ventilation maps $\left(\mathrm{V}_{100 \%}\right.$ map, $\mathrm{FV}_{100 \%}$ map), dynamic ventilation parameters (HVP, $\mathrm{V}\left(t_{\mathrm{x}}\right)$ curve, $\mathrm{FV}\left(t_{\mathrm{x}}\right)$ curve, and flow-volume loop), and $\mathrm{FV}_{\mathrm{Global}}$ for the healthy ventilation function of COVID-19 survivors. The results showed the static FD-V map could not distinguish survivors and healthy volunteers. However, the dynamic ventilation maps revealed lots of regional high ventilation areas existed in survivors. The regional heterogeneity marker (HVP) in survivors was significantly higher than that in healthy volunteers $(p=0.003)$. The survivors breathed deeper, breathed more air per unit time, and breathed more air in respiratory cycle. The proposed mPREFUL was validated by $\mathrm{FV}_{\mathrm{Globa}}$, showing both good Pearson's correlation and good consistency of the Bland-Altman plot.
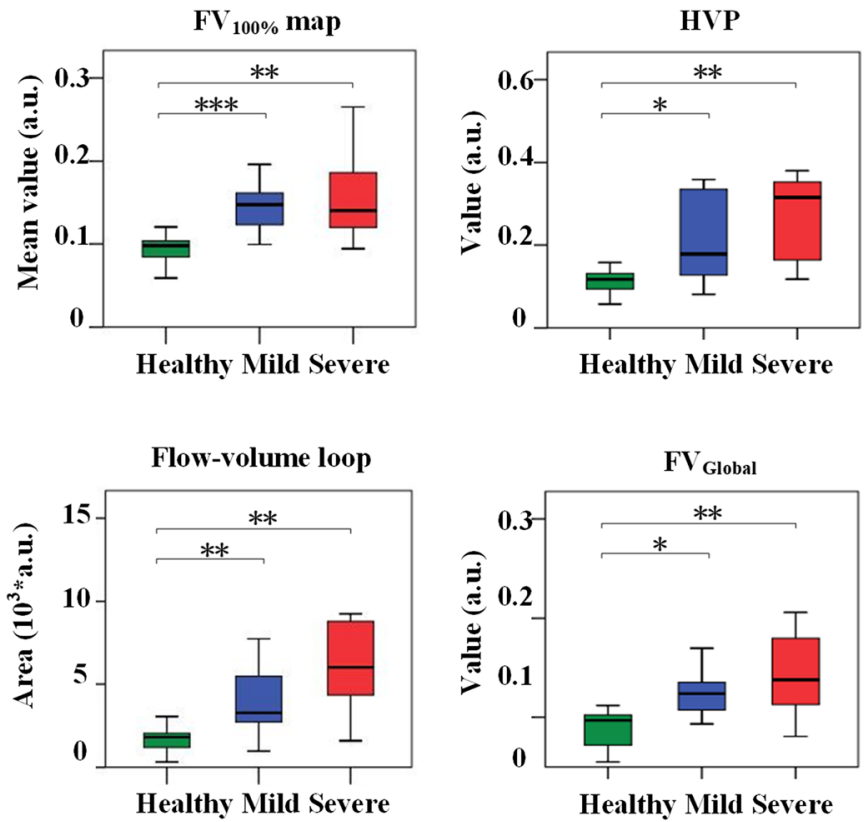

volunteers, mild COVID-19 survivors, and severe COVID-19 survivors. The symbol * meant $p<0.05$, ** meant $p<0.01$, and *** meant $p<$ 0.001 
a Measure relative change of lung volumes: $\left(V_{2}-V_{1}\right) / V_{2}$

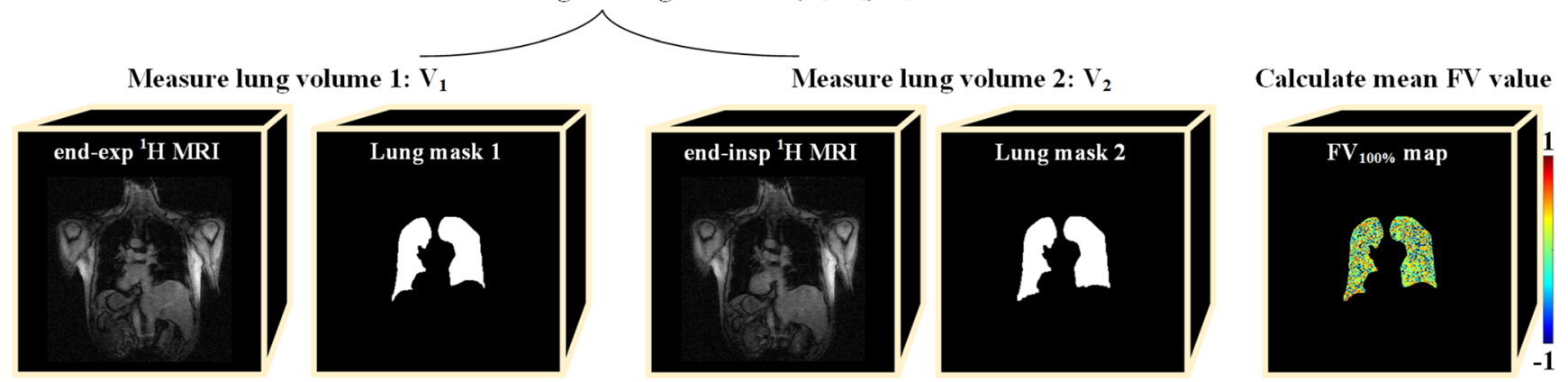

b

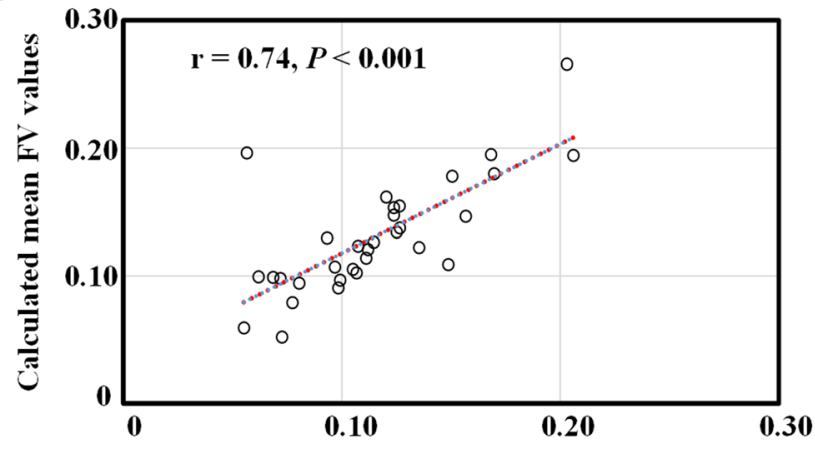

Measured relative changes of lung volumes

Fig. 6 The validation of dynamic ventilation maps. a The measurement of relative change between end-exp lung volume and end-insp lung volume. $\mathbf{b}$ The Pearson correlation between the measured relative changes of lung volumes and the calculated mean $\mathrm{FV}$ values of $\mathrm{FV}_{100 \%}$ maps for all

The dynamic ventilation maps were shown to be more sensitive to detect lung function abnormity in COVID-19 survivors, compared with static ventilation maps. The concept that dynamic ventilation imaging was more sensitive to detect lung function abnormity has also been demonstrated in recent PREFUL studies [36]. In this work, the value of dynamic ventilation imaging on the assessment of regional lung function in COVID-19 survivors was demonstrated.

The mPREFUL revealed the COVID-19 survivors breathed more air in respiratory cycle. Similarly, the $\mathrm{FV}_{\text {Global }}$ in survivors was also significantly higher than in healthy volunteers, which verified the mPREFUL findings. The global dynamic ventilation parameters could also be measured by PFTs. As reported, the tidal volume in the severe COVID-19 survivors (mean value $=1200 \mathrm{~mL}$ ) who were discharged about 35 days was much larger than that in the healthy adults (about $500 \mathrm{~mL}$ ) [5], which was also consistent with our findings. Nevertheless, PFTs could not provide regional information. Our study may provide some unique regional information, i.e., the regional dynamic ventilation abnormity, for the post COVID-19 researches. This regional information might be useful for interpreting the causes of lung dysfunction in COVID-19 survivors. For example, we found the increase of dynamic ventilation in COVID-19 survivors
C

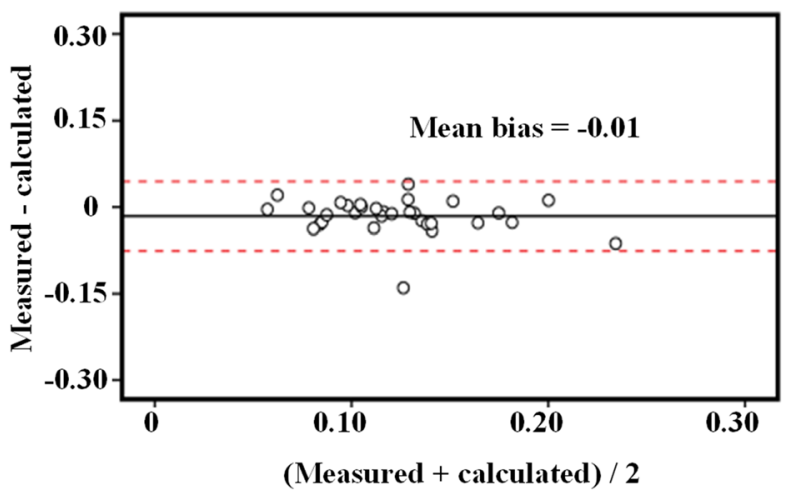

subjects. $\mathbf{c}$ The Bland-Altman plot of the measured relative changes of lung volumes and the calculated mean $\mathrm{FV}$ values of $\mathrm{FV}_{100 \%}$ maps for all subjects

was not uniformly distributed in the lungs but was concentrated in some regions of the lungs, which could not be observed in PFTs. Furthermore, the analysis on the regional information (e.g., the correlation analysis with CT residual lesions) would lead to the regional treatment on the lungs of COVID-19 survivors.

Previous studies [35-37, 44] have found regional high ventilation existed in some lung diseases (e.g., cystic fibrosis) and attributed this phenomenon as compensatory hyperventilation. The PFTs [7, 9] and hyperpolarized ${ }^{129}$ Xe MRI [19] studies have shown the impairment of diffusion capacity which would reduce the efficiency of oxygen entering blood was the most common lung dysfunction in COVID-19 survivors. In this study, the high dynamic ventilation was detected in COVID-19 survivors. A reasonable explanation was the compensatory mechanism of ventilation for the survivors, i.e., the bodies of survivors possibly self-adjusted to breathe more oxygen in respiratory cycle for exchanging with blood to maintain a normal arterial partial pressure of oxygen (as detected in this work). In future, that explanation should be more rigorously evaluated by measuring the perfusion or oxygen intake in survivors.

Our study has some limitations. First, sample size was relatively small. Besides, since this study was conducted during 
the outbreak period of the epidemic, some subjects felt nervous and cooperated not well. Thus, 1 healthy volunteer and 6 COVID-19 survivors were excluded because of image quality (e.g., motion artifacts). Second, only one coronal slice was acquired although this was a usual setting in free-breathing ${ }^{1} \mathrm{H}$ MRI [23]. However, the acquisition with more slices could image whole lung, which is needed in future. Third, detailed regional structural information was insufficient due to current limitation of free-breathing ${ }^{1} \mathrm{H}$ MRI. In the future, multiple modality imaging methods (such as CT, hyperpolarized ${ }^{129} \mathrm{Xe}$ MRI, and free-breathing ${ }^{1} \mathrm{H}$ MRI) should be developed to assess regional lung function of COVID-19 survivors.

In summary, the pulmonary free-breathing ${ }^{1} \mathrm{H}$ MRI was used to visualize and quantitatively assess regional lung ventilation function of COVID-19 survivors. The dynamic ventilation maps and parameters were more sensitive to distinguish the survivors and healthy volunteers, compared with static ventilation map. The dynamic ventilation imaging found the regional lung function abnormity in COVID-19 survivors. Pulmonary free-breathing ${ }^{1} \mathrm{H}$ MRI would be an optional method in clinical to examine regional lung function for the huge number of COVID-19 survivors to understand the longCOVID symptoms and identify the impairment where clinical features cannot.

Supplementary Information The online version contains supplementary material available at https://doi.org/10.1007/s00330-022-08605-w.

Acknowledgements Specially thanks to Dr. Sa Xiao for his help in this work.

Funding This work was supported by the National key Research and Development Project of China (2018YFA0704000), National Natural Science Foundation of China (91859206, 81625011, 21921004, 81825012, 82001915), Key Research Program of Frontier Sciences (ZDBS-LY-JSC004), and China Postdoctoral Science Foundation (2020M672454, 2020T130125ZX). Xin Zhou acknowledges the support from the Tencent Foundation through the XPLORER PRIZE.

\section{Declarations}

Guarantor The scientific guarantor of this publication is Xin Zhou.

Conflict of Interest The authors declare no competing interests.

Statistics and Biometry No complex statistical methods were necessary for this paper.

Informed Consent Written informed consent was obtained from all subjects (patients) in this study.

Ethical Approval Institutional Review Board approval was obtained.

\author{
Methodology \\ - prospective \\ - diagnostic or prognostic study \\ - performed at one institution
}

\section{References}

1. Zhu N, Zhang D, Wang W et al (2020) A novel coronavirus from patients with pneumonia in China, 2019. New Engl J Med 382(8): 727-733

2. World Health Organization (2021) Coronavirus disease (COVID2019) situation reports. Available via https://www.who.int/ publications $/ \mathrm{m} /$ item/weekly-operational-update-on-covid-19-30november-2021. Accessed 2 Dec 2021

3. Mo X, Jian W, Su Z et al (2020) Abnormal pulmonary function in COVID-19 patients at time of hospital discharge. Eur Respir J 55: 2001217

4. Hui DS, Joynt GM, Wong KT et al (2005) Impact of severe acute respiratory syndrome (SARS) on pulmonary function, functional capacity and quality of life in a cohort of survivors. Thorax 60:401-409

5. You J, Zhang L, Ni-Jia-Ti MY et al (2020) Anormal pulmonary function and residual $\mathrm{CT}$ abnormalities in rehabilitating COVID-19 patients after discharge. J Infection 81(2):e150-e152

6. Li X, Wang C, Kou S, Luo P, Zhao M, Yu K (2020) Lung ventilation function characteristics of survivors from severe COVID-19: a prospective study. Crit Care 24(1):300

7. Huang Y, Tan C, Wu J et al (2020) Impact of coronavirus disease 2019 on pulmonary function in early convalescence phase. Resp Res 21(1):163

8. Arnold DT, Hamilton FW, Milne A et al (2021) Patient outcomes after hospitalisation with COVID-19 and implications for followup: results from a prospective UK cohort. Thorax 76(4):399-401

9. Guler SA, Ebner L, Beigelman C et al (2021) Pulmonary function and radiological features four months after COVID-19: first results from the national prospective observational Swiss COVID-19 lung study. Eur Respir J 57(4):2003690

10. Pellegrino R, Viegi G, Brusasco, et al (2005) Interpretative strategies for lung function tests. Eur Respir J 26(5):948-968

11. King GG, Harris B, Mahadev S (2010) V/Q SPECT: utility for investigation of pulmonary physiology. Semin Nucl Med 40(6): 467-473

12. Cobes N, Guernou M, Lussato D et al (2020) Ventilation/perfusion SPECT/CT findings in different lung lesions associated with COVID-19: a case series. Eur J Nucl Med Mol I 47:10223

13. Kipritidis J, Tahir BA, Cazoulat G et al (2019) The VAMPIRE challenge: a multi-institutional validation study of CT ventilation imaging. Med Phys 46(3):1198-1217

14. Shao W, Patton TJ, Gerard SE et al (2019) N-phase local expansion ratio for characterizing out-of-phase lung ventilation. IEEE T Med Imaging 39(6):2025-2034

15. Wang C, Huang L, Xiao S et al (2021) Early prediction of lung lesion progression in COVID-19 patients with extended CT ventilation imaging. Eur J Nucl Med Mol I 48:4339-4349

16. Zhou X, Graziani D, Pines A (2009) Hyperpolarized xenon NMR and MRI signal amplification by gas extraction. Proc Natl Acad Sci U S A 106(40):16903-16906

17. Kauczor HU, Surkau R, Roberts T (1998) MRI using hyperpolarized noble gases. Eur Radiol 8(5):820-827

18. Li H, Zhang Z, Zhao X et al (2016) Quantitative evaluation of radiation-induced lung injury with hyperpolarized xenon magnetic resonance. Magn Reson Med 76(2):408-416

19. Li H, Zhao X, Wang Y, et al (2021) Damaged lung gas-exchange function of discharged COVID-19 patients detected by hyperpolarized ${ }^{129}$ Xe MRI. Sci Adv 7(1):eabc8180

20. Puderbach M, Eichinger M, Gahr J et al (2007) Proton MRI appearance of cystic fibrosis: comparison to CT. Eur Radiol 17(3):716724

21. Voskrebenzev A, Vogel-Claussen J (2021) Proton MRI of the lung: how to tame scarce protons and fast signal decay. J Magn Reson Imaging 53:1344-1357 
22. Zapke M, Topf H, Zenker $M$ et al (2006) Magnetic resonance lung function - a breakthrough for lung imaging and functional assessment? A phantom study and clinical trial. Resp Res 7(1):106

23. Bauman G, Puderbach M, Deimling M et al (2009) Non-contrastenhanced perfusion and ventilation assessment of the human lung by means of Fourier decomposition in proton MRI. Magn Reson Med 62(3):656-664

24. Bauman G, Lutzen U, Ullrich M et al (2011) Pulmonary functional imaging: qualitative comparison of Fourier decomposition MR Imaging with SPECT/CT in porcine lung. Radiology 260(2):551559

25. Bauman G, Scholz A, Rivoire J et al (2013) Lung ventilation- and perfusion-weighted Fourier decomposition magnetic resonance imaging: in vivo validation with hyperpolarized ${ }^{3} \mathrm{He}$ and dynamic contrast-enhanced MRI. Magn Reson Med 69(1):229-237

26. Pennati F, Quirk JD, Yablonskiy DA, Castro M, Aliverti A, Woods JC (2014) Assessment of regional lung function with multivolume ${ }^{1} \mathrm{H}$ MR imaging in health and obstructive lung disease: comparison with ${ }^{3} \mathrm{He}$ MR imaging. Radiology 273(2):580-590

27. Pennati F, Salito C, Borzani I et al (2019) Quantitative multivolume proton-magnetic resonance imaging in patients with cystic fibrosis lung disease: comparison with clinical indicators. Eur Respir J 53(5): 1702020

28. Capaldi DP, Eddy RL, Svenningsen S et al (2018) Free-breathing pulmonary MR imaging to quantify regional ventilation. Radiology 287(2): 171993

29. Capaldi DP, Guo F, Xing L, Parraga G (2020) Pulmonary ventilation maps generated with free-breathing proton MRI and a deep convolutional neural network. Radiology 298(2):202861

30. Guo F, Capaldi DP, Mccormack DG, Fenster A, Parraga G (2019) A framework for Fourier-decomposition free-breathing pulmonary ${ }^{1} \mathrm{H}$ MRI ventilation measurements. Magn Reson Med 81(3):2135-2146

31. Chassagnon G, Martin C, Marini R et al (2019) Use of elastic registration in pulmonary MRI for the assessment of pulmonary fibrosis in patients with systemic sclerosis. Radiology 291(2): 487-492

32. Heidenreich JF, Weng AM, Metz C et al (2020) Three-dimensional ultrashort echo time MRI for functional lung imaging in cystic fibrosis. Radiology 296(1):191-199

33. Marshall H, Deppe MH, Parra-Robles J et al (2011) Direct visualisation of collateral ventilation in COPD with hyperpolarised gas MRI. Thorax 67(7):613-617

34. Xiao S, Deng H, Duan C et al (2019) Highly and adaptively undersampling pattern for pulmonary hyperpolarized ${ }^{129} \mathrm{Xe}$ dynamic MRI. IEEE T Med Imaging 38(5):1240-1250

35. Voskrebenzev A, Gutberlet M, Klimes F et al (2018) Feasibility of quantitative regional ventilation and perfusion mapping with phase- resolved functional lung (PREFUL) MRI in healthy volunteers and COPD, CTEPH, and CF patients. Magn Reson Med 79(4):23062314

36. Alsady TM, Voskrebenzev A, Greer M et al (2019) MRI-derived regional flow-volume loop parameters detect early-stage chronic lung allograft dysfunction. J Magn Reson Imaging 50(6):18731882

37. Kaireit TF, Kern A, Voskrebenzev A et al (2021) Flow volume loop and regional ventilation assessment using phase-resolved functional lung (PREFUL) MRI: comparison with ${ }^{129}$ Xenon ventilation MRI and lung function testing. J Magn Reson Imaging 53(4):1092-1105

38. Pöhler GH, Klimeš F, Behrendt L et al (2021) Repeatability of phase-resolved functional lung (PREFUL)-MRI ventilation and perfusion parameters in healthy subjects and COPD patients. J Magn Reson Imaging 53:915-927

39. Klimeš F, Voskrebenzev A, Gutberlet $\mathrm{M}$ et al (2021) 3D phaseresolved functional lung ventilation MR imaging in healthy volunteers and patients with chronic pulmonary disease. Magn Reson Med 85(2):912-925

40. Voskrebenzev A, Gutberlet M, Becker L, Wacker F, VogelClaussen J (2016) Reproducibility of fractional ventilation derived by Fourier decomposition after adjusting for tidal volume with and without an MRI compatible spirometer. Magn Reson Med 76(5): $1542-1550$

41. National Health Commission of the People's Republic of China (2020) Guidelines for the diagnosis and treatment of novel coronavirus (2019-nCoV) infection (trial version 7). Available via https:// www.who.int/docs/default-source/wpro\%2D\%2D-documents/ countries/china/covid-19-briefing-nhe/1-clinical-protocols-for-thediagnosis-and-treatment-of-covid-19-v7.pdf?sfvrsn=c6cbfba4_2. Accessed 2 Dec 2021

42. Brookes GB, Fairfax AJ (1982) Chronic upper airway obstruction: value of the flow volume loop examination in assessment and management. J R Soc Med 75:425-434

43. Kirby M, Heydarian M, Svenningsen S et al (2012) Hyperpolarized ${ }^{3} \mathrm{He}$ magnetic resonance functional imaging semiautomated segmentation. Acad Radiol 19(2):141-152

44. Gouwens KR, Higano NS, Marks KT et al (2020) Magentic resonance imaging evaluation of regional lung Vts in severe neonatal bronchopulmonary dysplasia. Am J Resp Cirt Care Med 202(7): 1024-1031

Publisher's note Springer Nature remains neutral with regard to jurisdictional claims in published maps and institutional affiliations. 